\title{
Anterior capsular contraction after cataract surgery in eyes of diabetic patients
}

Satoshi Kato, Tetsuro Oshika, Jiro Numaga, Yoshie Hayashi, Miwako Oshiro, Takuma Yuguchi, Tadayoshi Kaiya

\begin{abstract}
Aim-To investigate change in the area of anterior capsular opening (ACO) after cataract surgery and its relation to the degree of postoperative anterior inflammation in patients with diabetes mellitus (DM).

Methods-31 eyes of 31 patients with DM and 30 eyes of 30 normal controls scheduled to undergo cataract surgery were examined prospectively. The area of ACO was measured with an anterior eye segment analysis system (EAS-1000) on the day following surgery and 3, 6, and 12 months after surgery. Comparative analyses were made on the area of ACO relative to the presence of $\mathrm{DM}$ and diabetic retinopathy (DR). The percentage reduction of area of ACO was calculated from values 1 day and 12 months after surgery, and multiple regression analysis was performed on the presence of DM, patient age, ACO area on the first postoperative day, and aqueous flare intensity 1 day and 12 months after surgery.
\end{abstract}

Results-The area was significantly smaller in the DM group at $3(p=0.015$, Student's $t$ test $), 6(p=0.011)$, and 12 $(p=0.010)$ months postoperatively. Patients having DR showed significantly smaller ACO area than the non-DR group $3(p=0.039), 6(p=0.033)$, and $12(p=0.028)$ months after surgery. Multiple regression analysis revealed that presence of $D M$ $(p=0.003)$ and aqueous flare intensity 12 months after surgery $(p=0.039)$ significantly correlated with the percentage reduction of area of ACO. Age, ACO area at 1 day postoperatively, and aqueous flare intensity immediately after surgery were not relevant to ACO contraction.

Conclusions-Anterior capsular contraction after cataract surgery was greater in eyes of DM patients, especially in those with DR and increased permeability of the blood-aqueous barrier.

(Brf Ophthalmol 2001;85:21-23)

Kaiya Eye Clinic

T Yuguchi

T Kaiya

Correspondence to:

Dr Satoshi Kato, Division of Ophthalmology, Tokyo

University Branch Hospital,

3-28-6 Mejirodai,

Bunkyo-ku, Tokyo, 112-8688

Japan

katou-s@ka2.so-net.ne.jp

Accepted for publication 16 May 2000 complications of CCC, including shrinkage and opacification of the anterior capsule, ${ }^{1-3}$ which interferes with postoperative fundus- copy of the peripheral retina, retinal photocoagulation, and vitreous surgery in case they are necessary. This is especially crucial in patients with diabetes mellitus (DM), since the presence of DM is considered to be a risk factor for the contraction of anterior capsular opening (ACO). ${ }^{4}$

Proliferation of the residual lens epithelial cells associated with increased collagen production is thought to be responsible for ACO contraction. ${ }^{5}$ It has been suggested that surgical invasion and contact with the IOL stimulate residual lens epithelial cells to produce cytokines such as interleukin 1 (IL-1), IL-6, IL-8, basic fibroblast growth factor, and transforming growth factor $\beta$. These cytokines may in turn affect the epithelial cells as autocrine and/or paracrine factors, and induce collagen production and fibrous proliferation. ${ }^{5}$ In parallel with these processes, aqueous prostaglandin $\mathrm{E}_{2}$ concentration is elevated, leading to damage of the blood-aqueous barrier and an increase in the aqueous protein concentration. ${ }^{6}$ Thus, the degree of postoperative inflammation may be related to the development of ACO contraction. Moreover, suggested risk factors of severe anterior capsular contraction include $\mathrm{DM},{ }^{4}$ pseudoexfoliation syndrome, ${ }^{7}$ pigmentary retinal degeneration, ${ }^{68}$ high myopia, ${ }^{9}$ advanced age, ${ }^{10}$ and uveitis. ${ }^{7}$ Many of these eyes have pre-existing deterioration of blood-aqueous barrier function, and/or are more predisposed to the barrier damage induced by surgical invasion. Therefore, it seems natural to assume a strong correlation between ACO contraction and anterior chamber inflammation, but there has been no study to confirm such an assumption.

This prospective study was undertaken to quantitatively assess the changes in ACO area after cataract surgery and to investigate the relation between ACO contraction and the degree of postoperative anterior chamber inflammation in patients with DM.

\section{Patients and methods}

Thirty one eyes of 31 consecutive DM patients (DM group, mean 68.9 (SD 8.3) years) and 30 eyes of 30 patients without DM (control group 69.5 (9.3) years), scheduled to undergo cataract surgery, were included in this study. If the patient was bilaterally treated, the eye which was first operated on was selected for the analysis. None of the eyes had any history of previous ocular surgery. Eyes were not included if they had pseudoexfoliation, pigmentary retinal degeneration, high myopia, history of trauma, or uveitis which are among the 


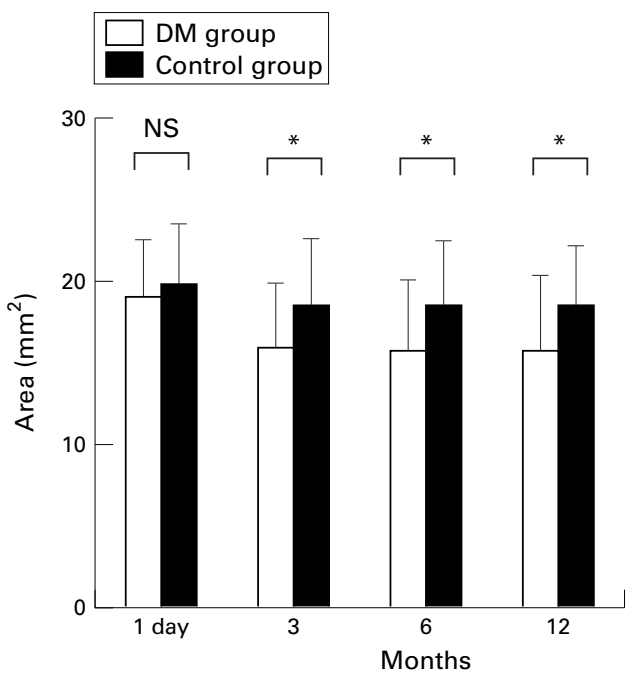

Figure 1 Area of anterior capsule opening in relation to the presence of diabetes mellitus. NS = not significant.

known risk factors of ACO shrinkage. Of the 31 diabetic eyes, 12 had diabetic retinopathy (DR group; 69.2 (4.9) years) and 19 had no retinopathy (NDR group; 68.7 (10.0) years). All eyes in the DR group had non-proliferative retinopathy. There was no significant difference in the mean age between the DM and control groups, and DR and NDR groups. All patients provided informed consent.

All cataract operations, consisting of phacoemulsification through a small incision and IOL implantation, were performed by one surgeon (TK). A CCC approximately $5.0 \mathrm{~mm}$ in diameter was created using a needle cystotome. After hydrodissection, phacoemulsification of the nucleus and cortical aspiration were performed. After the anterior chamber was filled with a viscoelastic agent, a foldable IOL with a $6.0 \mathrm{~mm}$ round optic (MA60BM; Alcon Surgical Inc) was inserted in the capsular bag. The viscoelastic agent was then washed out. One day postoperatively it was confirmed that the edge of CCC lay on the IOL optic completely, otherwise the eye was not included in the study population.

Postoperatively, all patients received similar routine medication, comprising topical application of diclofenac sodium, fluorometholone, and ofloxacin (antibacterial agent) four times daily for a month.

The aqueous flare intensity was measured using a laser flare cell meter (FC-1000 Kowa Co, Ltd) before surgery and 1 day, 3, 6, and 12 months after surgery. The flare intensity was measured 30-60 minutes after the application of $0.5 \%$ tropicamide and $0.5 \%$ phenylephrine hydrochloride (Mydrin P, Santen). Measurements were repeated 10 times and averaged to obtain final results for flare intensity. The area of ACO was determined by diaphanoscopy using the anterior eye segment analysis system EAS-1000 (Nidek, Inc) at 1 day, 3, 6, and 12 months postoperatively. The EAS- 1000 system is composed of a Scheimpflug charge coupled device video camera unit and an online image analysis computer. ${ }^{11}{ }^{12}$ The computer includes a software package to calculate the area of the

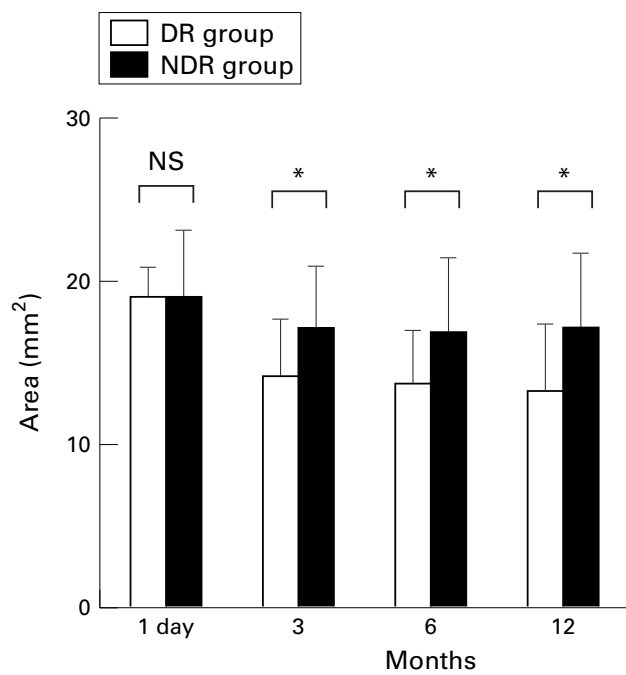

Figure 2 Area of anterior capsule opening in relation to the presence of diabetic retinopathy. ${ }^{\star} p<0.05$ (Student's $t$ test), NS = not significant.

eye's anterior segment that can also quantitate the area of ACO. ${ }^{8}$ If poor pupillary dilatation hindered the complete observation of the 360 degree anterior capsule margin, the patient was excluded from the study. The percentage reduction of area of ACO 12 months after cataract surgery was calculated as follows:

Percentage reduction of area of ACO $(\%)=$ ((ACO area 1 day after surgery - ACO area 12 months after surgery) $\times 100) / A C O$ area 1 day after surgery

Multiple regression analysis was performed to investigate the relation between various variables and the rate of ACO contraction at 12 months postoperatively. Variables tested included the presence of DM, patient age, ACO area on the first operative day, and aqueous flare intensity 1 day and 12 months after surgery.

\section{Results}

There was no significant difference in the mean area of ACO between the DM and control groups at 1 day postoperatively. Postoperatively, the DM group exhibited significant contraction of ACO (Fig 1). The ACO area in the DM group at $3(\mathrm{p}=0.00002$, paired $t$ test $), 6$ $(\mathrm{p}=0.000001)$, and $12(\mathrm{p}=0.00001)$ months postoperatively was significantly smaller than the value on the first operative day. The values in the control group did not fluctuate significantly throughout the study period. The ACO area in the DM group was significantly smaller than that of the control group at $3(\mathrm{p}=0.015$, Student's $t$ test $), 6 \quad(\mathrm{p}=0.011)$, and 12 $(\mathrm{p}=0.010)$ months postoperatively.

Figure 2 shows changes in the mean area of ACO in the DR and non-DR groups. There was no intergroup difference on the day following surgery, but the mean ACO area was significantly smaller in the DR group than in the non-DR group at $3 \quad(p=0.039), 6$ $(\mathrm{p}=0.033)$, and $12(\mathrm{p}=0.028)$ months postoperatively. In the DR group, the ACO area at 3 $(p=0.0009), 6(p=0.0006)$, and $12(p=0.001)$ months postoperatively was significantly 
Table 1 Results of multiple regression analysis to assess factors relevant to anterior capsule contraction 12 months after cataract surgery

\begin{tabular}{lll}
\hline & $\begin{array}{l}\text { Standardised regression } \\
\text { coefficient }\end{array}$ & p Value \\
\hline Presence of diabetes mellitus & 0.367 & 0.003 \\
Age & 0.150 & 0.213 \\
ACO area 1 day after surgery & -0.05 & 0.696 \\
Aqueous flare intensity 1 day after surgery & 0.046 & 0.721 \\
Aqueous flare intensity 1 year after surgery & 0.258 & 0.039 \\
\hline
\end{tabular}

$R^{2}=0.250$.

$\mathrm{ACO}=$ anterior capsule opening.

smaller than the value on the first operative day. In the non-DR group, such fluctuation was not observed.

The aqueous flare intensity before surgery and 1 day, 3, 6, and 12 months after surgery were 9.4 (SD 3.9) (photon counts $/ \mathrm{ms}$ ), 14.8 (11.1), 9.3 (3.7), 9.2 (4.3), $9.2(3.2)$ in the DM group and 9.0 (3.8), 13.7 (11.8), 8.9 (5.9), 8.4 (5.9), 8.3 (3.5) in the control group.

Multiple regression analysis showed that presence of DM $(p=0.003)$ and aqueous flare intensity 12 months after surgery $(p=0.027)$ were significantly correlated with the percentage reduction of area of ACO $\left(R^{2}=0.250\right.$, Table 1). Age at surgery, ACO area at 1 day postoperatively, and aqueous flare intensity immediately after surgery were not relevant to ACO contraction.

\section{Discussion}

In the present study, the percentage reduction of area of anterior capsule opening (ACO) was significantly greater in the diabetes mellitus (DM) group than in the control group. When compared in the DM group, patients with diabetic retinopathy exhibited more shrinkage of ACO than those without diabetic retinopathy. These findings are consistent with the results reported by Hayashi et $a l .{ }^{4}$ It has been also reported that significant capsular contraction occurred in diabetic eyes as early as 1 month after surgery. ${ }^{2}$ Severe shrinkage of the ACO will hamper the management and treatment of diabetic retinopathy. Thus, in consideration of the occurrence of ACO contraction, treatment such as retinal photocoagulation, if indicated after cataract surgery, should be carried out without delay. In addition, the current study revealed that no significant association exists between the original size of ACO and the percentage reduction of area of ACO. The percentage reduction of area of ACO being constant, attention should be given to the fact that a smaller capsulorhexis will result in a greater loss of the opening area. Creation of a relatively large $\mathrm{ACO}$ is recommended at the time of cataract surgery in patients with DM, especially in those having diabetic retinopathy. However, it should be noted that in the current study all eyes had the edge of the CCC stay on the IOL optic completely, and thus these arguments will not apply for the situation where the anterior capsular edge lies outside the IOL optic.

The current study showed that there is a significant correlation between aqueous flare intensity 12 months after surgery and the percentage reduction of area of ACO. On the other hand, flare intensity at 1 day postoperatively did not correlate with ACO contraction rate. Aqueous flare intensity immediately after surgery is thought to reflect the degree of operative invasion to the eye, while flare intensity in the late postoperative period represents the integrity of the blood-aqueous barrier. ${ }^{13}$ The current results indicate that more extensive ACO contraction occurs in eyes with increased permeability of the blood-aqueous barrier. The development of anterior capsular contraction after cataract surgery has been attributed to fibrous proliferation of residual lens epithelial cells with increased collagen production..$^{4-6}$ Postoperatively, residual lens epithelial cells are stimulated to produce cytokines, which may in turn affect the epithelial cells as autocrine and/or paracrine factors, inducing collagen production and fibrous proliferation. ${ }^{4}$ Prostaglandin $\mathrm{E}_{2}$, synthesised at the same time, may cause damage of the aqueousblood barrier, resulting in an elevation of flare intensity. ${ }^{5}$ Thus, it is assumed that the anterior capsular contraction and an elevation of flare intensity, as a result of the actions of cytokines produced following surgery, occurred simultaneously in the DM patients. Moreover, it has been known that DM patients are predisposed to the breakdown of the blood-aqueous barrier, ${ }^{14-16}$ and aqueous flare intensity increases in parallel to the progression of retinopathy..$^{15}$ These factors seem to have contributed to the extensive ACO contraction in patients with DM, especially in those with retinopathy.

1 Masket M. Postoperative complications of capsulorhexis. $\mathcal{F}$ Cataract Refract Surg 1993;19:721-4.

2 Kimura W, Yamanishi S, Kimura T, et al. Measurement the anterior capsule opening after cataract surgery to assess capsule shrinkage. F Cataract Refract Surg 1998;24:1235-8.

3 Sciscio A, Liu C. Anterior capsular phimosis following Acrysof lens insertion. Br f Ophthalmol 1999;83:989-90.

Acrysof lens insertion. Br f Ophthalmol 1999;83:989-90.
4 Hayashi H, Hayashi K, Nakao F, et al. Area reduction in the anterior capsule opening in eyes of diabetes mellitus anterior capsule opening in eyes of diabetes

5 Nishi T, Nishi K. Intraocular lens encapsulation by shrinkage of the capsulorhexis opening. F Cataract Refract Surg 1993;19:544-5

6 Nishi T, Nishi K. Fibrosis and constriction of capsular opening following continuous curvilinear capsulotomy. IOLERS (in Japanese) 1995;9:2-7.

7 Davison JA. Capsule contraction syndrome. 7 Cataract Refract Surg 1993;19:582-9.

8 Hayashi K, Hayashi H, Matsuo K, et al. Anterior capsule contraction and intraocular lens dislocation after implant contraction and intraocular lens dislocation after implant
surgery in eyes with retinitis pigmentosa. Ophthalmology 1998;105:1239-43.

9 Hansen SO, Crandall AS, Olson RJ. Progressive constriction of the anterior opening following intact capsulorhexis. $\mathcal{F}$ Cataract Refract Surg 1993;19:77-82.

10 Namiki M, Yamamoto N, Tagami Y, et al. Risk factors for anterior capsular shrinkage in intraocular lens implantation. Fpn f Clin Ophthalmol (in Japanese) 1991;45:1828-31.

11 Sasaki K, Sakamoto Y, Shibata T, et al. The multi-purpose camera;A new anterior eye segment analysis system. Ophthalmic Res 1990;22(suppl 1):3-8.

12 Sakamoto Y, Sasaki K. Accuracy of biometrical data obtained from the NIDEK EAS-1000. Ophthalmic Res 1994;26 (suppl 1):26-32.

13 Oshika T, Yoshimura K, Miyata N. Postsurgical inflammation after phacoemulsification and planned extracapsular extraction with soft or conventional intraocular lens implantation. F Cataract Refract Surg 1992;18:356-61.

14 Yoshida A, Ishiko S, Kojima M, et al. Permeability of the blood-ocular barrier in adolescent and adult diabetic patients. Brf Ophthalmol 1993;77:158-61.

15 Oshika T, Kato S, Funatsu H. Quantitative assessment of aqueous flare intensity in diabetes. Graefes Arch Clin Exp Ophthalmol 1989;227:518-20.

16 Moriarty AP, Spalton DJ, Moriarty BJ, et al. Studies of the blood-aqueous barrier in diabetes mellitus. Am $\mathcal{F}$ Ophthalmol 1994;117:768-71. 